\title{
Symbol-Level Precoding with Per-antenna Power Constraints for the Multi-beam Satellite Downlink
}

\author{
Danilo Spano*, Symeon Chatzinotas*, Jens Krause ${ }^{\dagger}$, and Björn Ottersten* \\ ${ }^{*} \mathrm{SnT}$ - securityandtrust.lu, University of Luxembourg \\ email: \{danilo.spano, symeon.chatzinotas, bjorn.ottersten\}@uni.lu \\ ${ }^{\dagger}$ SES, Chateau de Betzdorf, Luxembourg, email: jens.krause@ses.com
}

\begin{abstract}
This paper tackles the problem of multi-user interference in the forward downlink channel of a multi-beam satellite system. A symbol-level precoding scheme is considered, where the data information is used, along with the channel state information, in order to exploit the multi-user interference and transform it into useful power at the receiver side. In this framework, the max-min fair problem for constructive interference is formulated and solved, under per-antenna power constraints. The consideration of the power limitations individually for each transmitting RF chain is the novel aspect, and it is relevant in particular for systems suffering non-linear effects of the channel. This is the case of satellite systems, where the nonlinear amplifiers should be properly driven in order to reduce the detrimental saturation effect. The proposed precoding design optimizes the system performance at the receiver side in terms of signal-to-noise ratio, whilst guaranteeing the system fairness and allowing a control over the power transmitted by each antenna, in particular reducing the power peaks. Numerical results are presented to validate the proposed scheme, and to show its effectiveness in terms of distribution of the transmitted power and peak-to-average power ratio.
\end{abstract}

\section{INTRODUCTION}

In the current research on satellite communication (SatCom) systems, one of the biggest challenges is the need to break the existent throughput gridlock, in order to fulfill the everincreasing demand for interactive services and multimedia content delivery. The state of the art in high throughput SatComs relies on multi-beam architectures, which exploit the spatial degrees of freedom offered by antenna arrays to aggressively reuse the available spectrum, thus realizing a space division multiple access (SDMA) scheme [1]. In this framework, the ongoing research work is focused on developing advanced signal processing techniques, capable of managing the multi-user interference (MUI) arising in multiantenna systems and limiting the performance. These techniques are commonly referred to as multi-user multiple-input multiple-output (MU-MIMO) and, in the satellite context, also as multi-beam joint processing. Multi-beam joint processing enables a more aggressive frequency reuse, thus enhancing the overall throughput of next generation SatCom networks, which strive for terabit capacity [2]-[6]. In this context, linear precoding (or beamforming) has been shown to be an effective way to manage the MUI while guaranteeing some specific service requirements [7]-[12]. The benefits of using precoding techniques for managing the interference at the gateway in
SatComs are also considered in the most recent extensions of broadband multi-beam SatCom standards [13].

The conventional precoding approach exploits the knowledge of the channel state information (CSI) in order to design a precoder to be applied to the multiple data streams, thus mitigating the MUI. Therefore, this scheme is also known as channel-level precoding. Different strategies have been considered in the literature for the precoder design. The optimal precoding algorithm for the minimization of the total transmit power, whilst guaranteeing some Quality-of-Service (QoS) targets at each user, was given in [10], [12], while the max-min fair problem under sum power constraints (SPC) was optimally solved in [11]. The latter strategy aims to maximize the minimum signal-to-interference-plus-noise ratio (SINR) amongst the users, in order to preserve the fairness of the system. The reaserch work on channel-level precoding was extended in [14] accounting for per-antenna power constraints (PACs), and in [15], [16] considering generalized power constraints. Furthermore, the problem of channel-level precoding in a multigroup multicast framework has been tackled in [17].

A different precoding strategy, considered more recently in the literature, is known as symbol-level precoding [18]-[23]. In this approach, the transmitted signals are designed based on the knowledge of both the CSI and the data information (DI), constituted by the symbols to be delivered to the users. Since the design exploits also the DI, the objective of symbollevel precoding is not to eliminate the interference, but rather to control it so to have a constructive interference (CI) effect at each user. The classification of the interference as constructive or destructive was given in [18], where a selective channel inversion scheme was proposed in order to eliminate the destructive interference. A more advanced symbol-level precoding scheme was proposed in [19], based on the rotation of the destructive interference, with the aim to transform it into useful power. Different optimization approaches have been proposed in the literature for symbol-level precoding. In [22] the sum-power minimization and the max-min fair problem were solved for M-PSK modulations. Furthermore, symbol-level precoding has been considered for multicastbased systems and for multi-level modulations, including also flexible schemes accounting an imperfect knowledge of the CSI, as well as relaxed detection regions [20], [21], [23].

In this work, the problem of symbol-level precoding is addressed taking into account the per-antenna power limitations 
of the transmitter, which have not been tackled in the existent literature. In particular a symbol-level max-min fair scheme, with PACs, is proposed and solved for M-PSK modulations. The need of per-antenna constraints is usually motivated by the practical implementation of systems that rely on precoding. In fact, a common practice in multi-antenna systems is the use of individual per-antenna amplifiers, and this implies a lack of flexibility in sharing energy resources amongst the antennas of the transmitter. In spite of the possibility of using flexible amplifiers so to handle this issue, specific communication systems cannot afford this design. Typical per-antenna power limited systems can be found in multi-beam SatComs [24], where flexible on-board payloads are difficult to implement.

An additional important challenge in SatComs is the need to counteract the non-linear effects usually introduced by the onboard per-antenna traveling-wave-tube amplifiers (TWTAs), which result in a distortion on the transmitted waveforms [13], [25]-[27]. A typical solution to this problem in single-user links relies on predistortion techniques [25], [28], [29], but their extension to multi-beam systems relying on precoding is not straightforward, because of the mutual correlation between the data streams induced by the precoding schemes. About this, a joint predistortion algorithm for multi-beam systems is given in [30]. In this framework, the proposed precoding scheme allows to mitigate the signal corruption induced by the non-linear satellite channel. In fact, the combination of a symbol-level design with the use of PACs allows to have a control on the instantaneous per-antenna transmit power, and in particular to keep the power peaks in the linear region of the TWTA.

The remainder of the paper is organized as follows. In Section 2, the system and signals communication model is delineated. In Section 3, the symbol-level max-min fair problem with PACs is proposed and solved. In Section 4, the proposed approach is validated through simulation results. Finally, in Section 5 conclusions are drawn.

Notation: We use upper-case and lower-case bold-faced letters to denote matrices and vectors, respectively. $(\cdot)^{T}$ denotes the transpose operator. $|\cdot|$ and $\angle(\cdot)$ denote the amplitude and the phase of $(\cdot)$, respectively, while $\operatorname{Re}(\cdot)$ and $\operatorname{Im}(\cdot)$ are the real and imaginary parts of $(\cdot) .\|\cdot\|$ and $\|\cdot\|_{\infty}$ represent the Euclidean norm and the $1_{\infty}$ norm of $(\cdot)$, respectively. $\operatorname{Pr}(\cdot)$ denotes the probability of an event, while $\mathbb{E}_{n}(\cdot)$ denotes the statistical expectation evaluated with respect to the index $n$. Finally, $\operatorname{diag}(\cdot)$ denotes a diagonal matrix whose diagonal entries are the elements of $(\cdot)$, while $\circ$ is used for denoting the element-wise Hadamard operations.

\section{System And Signals Model}

We focus on a multi-user (MU) multiple-input singleoutput (MISO) satellite system. Let $N_{t}$ denote the number of transmitting elements of the transmitter and $K$ the number of users, with $K \leq N_{t}$, each one equipped with a single receiving antenna. The adopted modulation is M-PSK, and a channel vector $\boldsymbol{h}_{j} \in \mathbb{C}^{1 \times N_{t}}$ is assumed between the transmitting antennas and the $j$-th user. The received signal at the $j$-th user in the symbol slot $n$ can be written as:

$$
y_{j}[n]=\boldsymbol{h}_{j} \boldsymbol{x}[n]+z_{j}[n],
$$

where $\boldsymbol{x}[n] \in \mathbb{C}^{N_{t} \times 1}$ represents the transmitted signal vector from the $N_{t}$ transmit antennas, and $z_{j}[n]$ is a complex circular symmetric random variable, modeling the zero mean Additive White Gaussian Noise (AWGN) measured at the $j$-th user's receiving antenna. Without loss of generality, the noise variance is assumed to be 1 .

By collecting the received signals by all the users in a vector $\boldsymbol{y}[n] \in \mathbb{C}^{K \times 1}$, the above model can be rewritten in a compact form as:

$$
\boldsymbol{y}[n]=\boldsymbol{H} \boldsymbol{x}[n]+\boldsymbol{z}[n],
$$

where $\boldsymbol{H}=\left[\boldsymbol{h}_{1}^{T} \ldots \boldsymbol{h}_{K}^{T}\right]^{T} \in \mathbb{C}^{K \times N_{t}}$ represents the system channel matrix, and $\boldsymbol{z}[n] \in \mathbb{C}^{K \times 1}$ collects the AWGN components for all the users.

As regard to the channel model, assuming fixed users with highly directive antennas, we consider real channel gains depending only on the multi-beam antenna pattern and on the users position ${ }^{1}$. Considering the $i$-th beam and the $k$-th user, the corresponding entry of the channel matrix $\boldsymbol{H}$ can be calculated resorting to the well accepted method of Bessel functions, thus it will be the square root of the following power gain [31]:

$$
g_{i k}\left(\theta_{i k}\right)=G_{\max }\left(\frac{J_{1}(u)}{2 u}+36 \frac{J_{3}(u)}{u^{3}}\right)^{2},
$$

where $\theta_{i k}$ is the off-axis angle of the user with respect to the boresight of the beam, $u=2.07123 \sin \theta_{i k} / \sin \theta_{3 \mathrm{~dB}}$, with $\theta_{3 \mathrm{~dB}}$ being the one-sided half-power angular beamwidth, $G_{\max }$ is the maximum on-axis power gain of the antenna, and $J_{1}$, $J_{3}$ are the Bessel functions of the first kind, of order one and three respectively.

According to the symbol-level precoding approach [22], the transmitted signal vector $\boldsymbol{x}[n]$ is obtained as output of a precoding module, which directly designs $\boldsymbol{x}[n]$ using the CSI, which is an estimate of $\boldsymbol{H}$, and the input data symbols $\boldsymbol{d}[n] \in \mathbb{C}^{K \times 1}$, namely the DI that the BS wants to convey to the users. The data symbols, drawn from an M-PSK constellation, are assumed to be uncorrelated and having unit power. It should be noticed that, after the precoding operation, the constellation diagram of the transmitted signals is completely different from the M-PSK one associated to the original DI, because of the correlation between the multiple data streams induced by the precoding module. To better illustrate this, referring to an example with 7 beams and a 8-PSK modulation for the DI, Figs. 1-2 show the scatter plot of a large number of symbols associated to one of the transmitting antennas, before and after the application of precoding (the max-min fair symbol-level scheme with SPC of [22] is used), respectively.

\footnotetext{
${ }^{1}$ However, the main conclusions of this work are still valid if a random phase is incorporated in the channel model.
} 


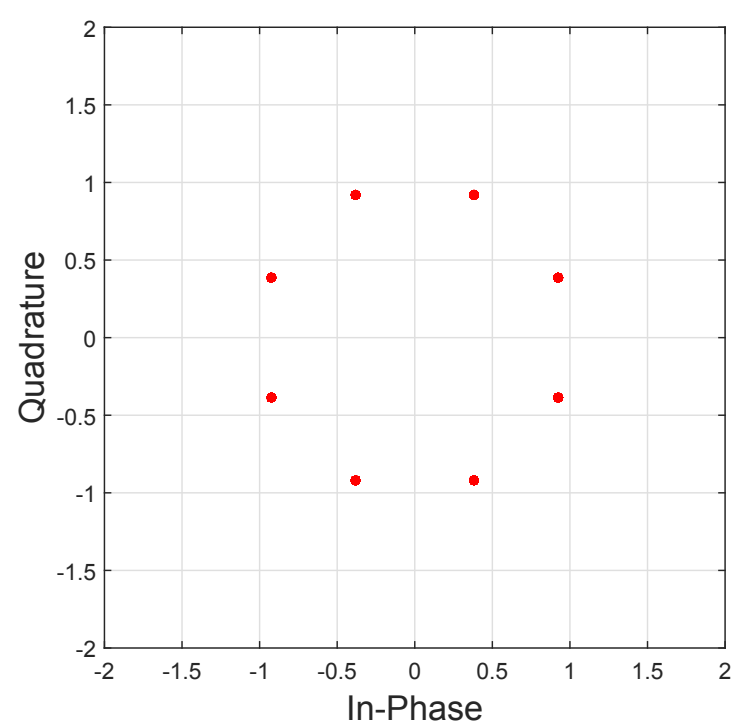

Figure 1: Scatter plot of the symbols (8-PSK) associated to one antenna, before precoding.

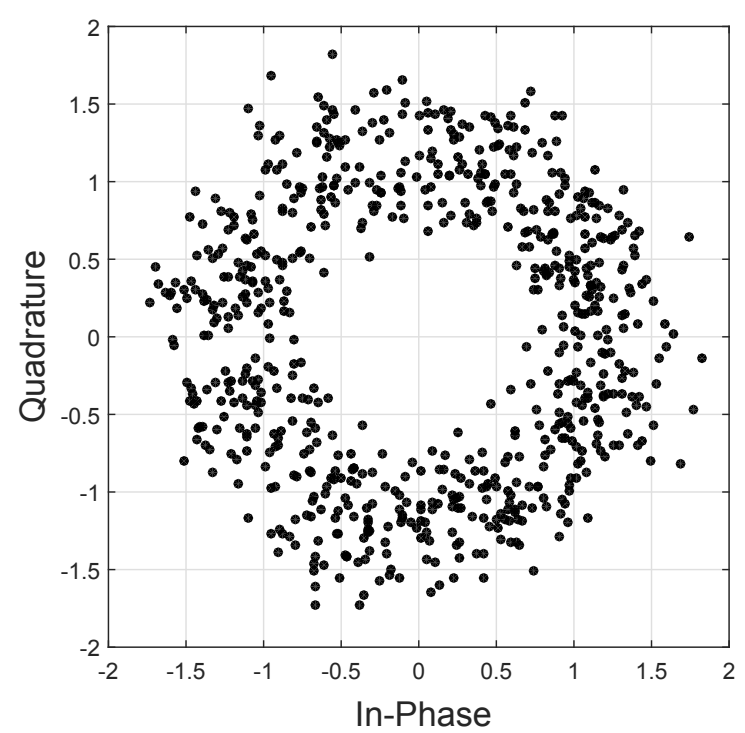

Figure 2: Scatter plot of the symbols associated to one antenna, after symbol-level precoding with SPC.

The effect of precoding is clearly visible, in particular with respect to the transmitted power, which is far from being constant for the various symbols and actually presents some peaks.

As already mentioned, it should be considered that the system model of (2) is actually corrupted by the non-linear effects introduced by the on-board per-antenna TWTAs, which affect both the amplitude and the phase of the transmitted waveforms. Different models describing the relationship between the input and the output signals of the amplifiers are provided in [13], [27]. Fig. 3 shows the normalized amplitude-

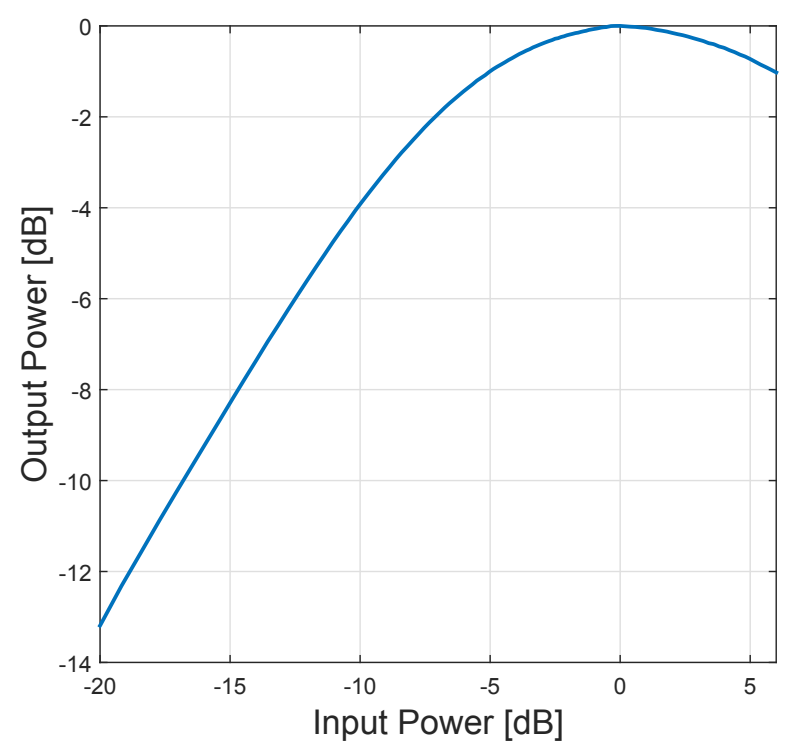

Figure 3: Normalized AM-AM characteristic of the on-board TWTAs (non-linearized model).

to-amplitude (AM-AM) characteristic of the common nonlinearized TWTA model, where the saturation effect introduced by the amplifier can be noticed. The on-board TWTAs need to be operated as close as possible to saturation, to efficiently exploit the scarce available power. As a consequence, the need of controlling the power level of the transmitted waveforms is pivotal in order to reduce the detrimental effect of the non-linearities of the satellite channel, which is critical in presence of precoding, because of the complex structure of the transmitted constellations. In particular, the transmitted power should be kept below the saturation point of the amplifiers.

\section{Symbol-Level Precoding With Per-Antenna POWER CONSTRAINTS}

\section{A. Constructive Interference Max-Min Fair with Per-antenna Power Constraints (CIMM-PAC)}

The aim is to design the transmitted vector $\boldsymbol{x}$ (to ease the notation, hereafter the time index $n$ is omitted), based on the CSI and the DI, assuring that the received signal lies in the detection region of the desired symbol, for each user. This way, the interfering signals are forced to constructively contribute to the useful received power, in line with the definition of constructive interference provided in [22]. More specifically, the proposed approach aims to maximize the minimum SINR amongst the users (max-min fair), while satisfying some perantenna constraints for the transmitted power, in addition to the constructive interference condition. The use of PACs is the novel aspect of the work, with respect to the max-min fair problem solved in [22], where the imposed constraints are over the total transmitted power (hence a SPC is considered). The resulting optimization problem, which can be referred to 
as constructive interference max-min fair with PACs (CIMM$\mathrm{PAC})$, is the following:

$$
\begin{aligned}
\boldsymbol{x}(\boldsymbol{d}, \boldsymbol{H})= & \arg \max _{\boldsymbol{x}} \min _{j=1, \ldots, K}\left\{\left|\boldsymbol{h}_{j} \boldsymbol{x}\right|^{2}\right\} \\
& \text { subject to } \\
& \left|x_{i}\right|^{2} \leq P_{\mathrm{Th}}, i=1, \ldots, N_{t}, \\
& \angle \boldsymbol{h}_{j} \boldsymbol{x}=\angle d_{j}, j=1, \ldots, K .
\end{aligned}
$$

The first set of constraints represent the PACs, imposing that the power transmitted by each antenna should be not larger than a predefined threshold power $P_{\mathrm{Th}}$. The second set of constraints represents the constructive interference condition, guaranteeing that each user receives the desired data symbol.

With respect to the max-min fair problem with SPC of [22] (which hereafter will be referred to as CIMM-SPC), we can expect worse performance in terms of attained SINR for a linear system, because of the tighter constraints. However, if the non-linearities of the satellite channel are taken into account, the proposed scheme with PACs can present some advantages. In particular, a wise choice of the value of $P_{\mathrm{Th}}$, in relation to the saturation power of the on-board TWTAs, can guarantee that even the power peaks of the transmitted waveforms (visible in the example of Fig. 2) lie in the linear region of the amplifiers. For instance, denoting by $P_{\text {Sat }}$ the saturation power of the amplifiers, a possible choice for the PACs could be $P_{\mathrm{Th}}^{\mathrm{dB}}=P_{\mathrm{Sat}}^{\mathrm{dB}}-\Delta^{\mathrm{dB}}$, where the values are in $\mathrm{dB}$ and $\Delta$ denotes an imposed minimum separation interval.

The problem (4) can be solved, in the same fashion of [22], based on a bisection procedure on the solution of the equivalent power minimization problem. The related per-antenna power minimization problem is addressed and solved by the authors in [32]. Hereinafter, for the sake of completeness, the problem formulation and the solution derivation are recalled. Then, the relation between the power minimization problem and the max-min fair problem at hand is discussed.

\section{B. Constructive Interference for Peak-Power Minimization (CIPPM)}

The problem of symbol-level precoding for per-antenna power minimization, under QoS constraints, is solved in [32]. The proposed formulation aims at minimizing the maximum power among the different transmitting antennas, hence the problem is referred to as constructive interference for peakpower minimization (CIPPM). The CIPPM problem reads as:

$$
\begin{aligned}
\boldsymbol{q}(\boldsymbol{d}, \boldsymbol{H}, \gamma)= & \arg \min _{\boldsymbol{q}} \max _{i=1, \ldots, N_{t}}\left\{\left|q_{i}\right|^{2}\right\} \\
& \text { subject to } \\
& \left|\boldsymbol{h}_{j} \boldsymbol{q}\right|^{2} \geq \gamma_{j}, j=1, \ldots, K, \\
& \angle \boldsymbol{h}_{j} \boldsymbol{q}=\angle d_{j}, j=1, \ldots, K,
\end{aligned}
$$

where $\boldsymbol{q}$ denotes the transmitted signal vector ${ }^{2}, \gamma_{j}$ is the target SINR that should be granted for the $j$-th user, and $\gamma=\left[\gamma_{1} \ldots \gamma_{K}\right]^{T} \in \mathbb{C}^{K \times 1}$ stacks the target SINR for all

${ }^{2}$ To avoid ambiguity, we are using a different notation for the solution to the CIPPM problem $(\boldsymbol{q})$ and the solution to the CIMM-PAC problem $(\boldsymbol{x})$. the users. Below, the steps followed in [32] for solving the problem are briefly retraced.

First of all, following the method of [33], by defining $\alpha_{j}=\tan \left(\angle d_{j}\right) \forall j=1, \ldots, K$, the equality and inequality constraints can be rewritten, respectively, as:

$$
\frac{\operatorname{Im}\left(\boldsymbol{h}_{j} \boldsymbol{q}\right)}{\operatorname{Re}\left(\boldsymbol{h}_{j} \boldsymbol{q}\right)}=\alpha_{j}, j=1, \ldots, K
$$

and

$$
\begin{aligned}
& \operatorname{Re}\left(d_{j}\right) \operatorname{Re}\left(\boldsymbol{h}_{j} \boldsymbol{q}\right) \geq \sqrt{\gamma_{j}} \operatorname{Re}^{2}\left(d_{j}\right), j=1, \ldots, K \\
& \operatorname{Im}\left(d_{j}\right) \operatorname{Im}\left(\boldsymbol{h}_{j} \boldsymbol{q}\right) \geq \sqrt{\gamma_{j}} \operatorname{Im}^{2}\left(d_{j}\right), j=1, \ldots, K
\end{aligned}
$$

With these new constraints, and resorting to the concept of $1_{\infty}$-norm, the CIPPM problem can be rewritten as:

$$
\begin{aligned}
& \boldsymbol{q}(\boldsymbol{d}, \boldsymbol{H}, \boldsymbol{\gamma})=\arg \min _{\boldsymbol{q}}\|\boldsymbol{q}\|_{\infty} \\
& \text { subject to } \\
& \operatorname{Re}\left(d_{j}\right) \operatorname{Re}\left(\boldsymbol{h}_{j} \boldsymbol{q}\right) \geq \sqrt{\gamma_{j}} \operatorname{Re}^{2}\left(d_{j}\right), j=1, \ldots, K, \\
& \operatorname{Im}\left(d_{j}\right) \operatorname{Im}\left(\boldsymbol{h}_{j} \boldsymbol{q}\right) \geq \sqrt{\gamma_{j}} \operatorname{Im}^{2}\left(d_{j}\right), j=1, \ldots, K, \\
& \frac{\operatorname{Im}\left(\boldsymbol{h}_{j} \boldsymbol{q}\right)}{\operatorname{Re}\left(\boldsymbol{h}_{j} \boldsymbol{q}\right)}=\alpha_{j}, j=1, \ldots, K
\end{aligned}
$$

and, in a more compact form, as follows:

$$
\begin{aligned}
\boldsymbol{q}(\boldsymbol{d}, \boldsymbol{H}, \boldsymbol{\gamma})= & \arg \min _{\boldsymbol{q}}\|\boldsymbol{q}\|_{\infty} \\
& \text { subject to } \\
& \operatorname{Re}(\boldsymbol{D}) \operatorname{Re}(\boldsymbol{H} \boldsymbol{q}) \geq \boldsymbol{\beta}_{\boldsymbol{r}} \\
& \operatorname{Im}(\boldsymbol{D}) \operatorname{Im}(\boldsymbol{H} \boldsymbol{q}) \geq \boldsymbol{\beta}_{\boldsymbol{i}} \\
& \boldsymbol{A} \operatorname{Re}(\boldsymbol{H} \boldsymbol{q})-\operatorname{Im}(\boldsymbol{H} \boldsymbol{q})=\mathbf{0}
\end{aligned}
$$

where $\boldsymbol{D}=\operatorname{diag}(\boldsymbol{d}), \boldsymbol{A}=\operatorname{diag}\left(\alpha_{1}, \ldots, \alpha_{K}\right), \boldsymbol{\beta}_{\boldsymbol{r}}=\sqrt{\boldsymbol{\gamma}} \circ$ $\operatorname{Re}(\boldsymbol{d})^{\circ 2}, \boldsymbol{\beta}_{\boldsymbol{i}}=\sqrt{\boldsymbol{\gamma}} \circ \operatorname{Im}(\boldsymbol{d})^{\circ 2}$.

The problem (9) can be written as a second-order cone programming (SOCP) [34] in the stacked variable $\tilde{\boldsymbol{q}}=$ $\left[\operatorname{Re}(\boldsymbol{q})^{T}, \operatorname{Im}(\boldsymbol{q})^{T}\right]^{T} \in \mathbb{R}^{2 N_{t} \times 1}$. To this end, the objective function should be written as:

$$
\|\boldsymbol{q}\|_{\infty}=\max _{i=1, \ldots, N_{t}}\left\{\left|q_{i}\right|\right\}=\max _{i=1, \ldots, N_{t}}\left\|\boldsymbol{B}_{i} \tilde{\boldsymbol{q}}\right\|
$$

where $\boldsymbol{B}_{i} \in \mathbb{R}^{2 \times 2 N_{t}}$ is a matrix used for selecting $\operatorname{Re}\left(q_{i}\right)$ and $\operatorname{Im}\left(q_{i}\right)$ in the stacked vector $\tilde{\boldsymbol{q}}$ and, $\forall i=1, \ldots, N_{t}$, is in turn defined as:

$$
\left[\begin{array}{cc}
\boldsymbol{e}_{i} & \mathbf{0}_{N_{t}}^{T} \\
\mathbf{0}_{N_{t}}^{T} & \boldsymbol{e}_{i}
\end{array}\right]
$$

with $\boldsymbol{e}_{i}$ being a the $i$-th row of an identity matrix with size $N_{t}$, and $\mathbf{0}_{N_{t}}$ being the all zero entries vector in $\mathbb{R}^{N_{t} \times 1}$.

By defining the $\boldsymbol{H}_{\mathbf{1}}=[\operatorname{Re}(\boldsymbol{H}),-\operatorname{Im}(\boldsymbol{H})]$ and $\boldsymbol{H}_{\mathbf{2}}=$ $[\operatorname{Im}(\boldsymbol{H}), \operatorname{Re}(\boldsymbol{H})]$, the problem (9) becomes: 


$$
\begin{aligned}
\tilde{\boldsymbol{q}}(\boldsymbol{d}, \boldsymbol{H}, \boldsymbol{\gamma})= & \arg \min _{\tilde{\boldsymbol{q}}} \max _{i=1, \ldots, N_{t}}\left\|\boldsymbol{B}_{i} \tilde{\boldsymbol{q}}\right\| \\
& \text { subject to } \\
& \operatorname{Re}(\boldsymbol{D}) \boldsymbol{H}_{\mathbf{1}} \tilde{\boldsymbol{q}} \geq \boldsymbol{\beta}_{\boldsymbol{r}}, \\
& \operatorname{Im}(\boldsymbol{D}) \boldsymbol{H}_{\mathbf{2}} \tilde{\boldsymbol{q}} \geq \boldsymbol{\beta}_{\boldsymbol{i}}, \\
& \left(\boldsymbol{A} \boldsymbol{H}_{\mathbf{1}}-\boldsymbol{H}_{\mathbf{2}}\right) \tilde{\boldsymbol{q}}=\mathbf{0} .
\end{aligned}
$$

Finally, by introducing a slack variable $r$, the CIPPM problem can be formulated as a SOCP as follows:

$$
\begin{aligned}
\tilde{\boldsymbol{q}}(\boldsymbol{d}, \boldsymbol{H}, \boldsymbol{\gamma})= & \arg \min _{r, \tilde{\boldsymbol{q}}} \quad r \\
& \text { subject to } \\
& \left\|\boldsymbol{B}_{i} \tilde{\boldsymbol{q}}\right\| \leq r, i=1, \ldots, N_{t}, \\
& \operatorname{Re}(\boldsymbol{D}) \boldsymbol{H}_{\mathbf{1}} \tilde{\boldsymbol{q}} \geq \boldsymbol{\beta}_{\boldsymbol{r}} \\
& \operatorname{Im}(\boldsymbol{D}) \boldsymbol{H}_{\mathbf{2}} \tilde{\boldsymbol{q}} \geq \boldsymbol{\beta}_{\boldsymbol{i}} \\
& \left(\boldsymbol{A} \boldsymbol{H}_{\mathbf{1}}-\boldsymbol{H}_{\mathbf{2}}\right) \tilde{\boldsymbol{x}}=\mathbf{0} .
\end{aligned}
$$

This optimization problem can be efficiently solved using the standard convex optimization tools.

\section{Relation between the CIMM-PAC and the CIPPM Prob- lems}

The relation between the max-min fair and the power minimization problems can be established, in the same fashion of [22], rewriting the CIMM-PAC problem (4) as follows, where the slack variable $t$ in introduced:

$$
\begin{aligned}
\boldsymbol{x}(\boldsymbol{d}, \boldsymbol{H})= & \arg \max _{t, \boldsymbol{x}} t \\
& \text { subject to } \\
& \left|x_{i}\right|^{2} \leq P_{\mathrm{Th}}, i=1, \ldots, N_{t}, \\
& \angle \boldsymbol{h}_{j} \boldsymbol{x}=\angle d_{j}, j=1, \ldots, K, \\
& \left|\boldsymbol{h}_{j} \boldsymbol{x}\right|^{2} \geq t, j=1, \ldots, K .
\end{aligned}
$$

Denoting by $t^{*}$ the optimal value of $t$ in the problem (14), it turns out that the relation between the CIMM-PAC and the CIPPM problems can be described as $\boldsymbol{x}(\boldsymbol{d}, \boldsymbol{H})=$ $\boldsymbol{q}\left(\boldsymbol{d}, \boldsymbol{H}, t^{*} \mathbf{1}_{K}\right)$, where $\mathbf{1}_{K}$ denotes the all one entries vector in $\mathbb{R}^{K \times 1}$. In other words, the max-min fair solution is a scaled version of minimum power solution. This implies that the solution to the CIMM-PAC problem can be found applying a simple bisection procedure [34] over the solution of the equivalent CIPPM problem, as in [22].

\section{Numerical RESUlts}

In this section some numerical results are presented, in order to validate the proposed CIMM-PAC approach. For the sake of comparison, we consider also numerical results related to the CIMM-SPC scheme of [22], whose corresponding optimization problem is the following:

$$
\begin{aligned}
\boldsymbol{x}(\boldsymbol{d}, \boldsymbol{H})= & \arg \max _{\boldsymbol{x}} \min _{j=1, \ldots, K}\left\{\left|\boldsymbol{h}_{j} \boldsymbol{x}\right|^{2}\right\} \\
& \text { subject to } \\
& \|\boldsymbol{x}\|^{2} \leq P_{\text {Tot }}, \\
& \angle \boldsymbol{h}_{j} \boldsymbol{x}=\angle d_{j}, j=1, \ldots, K,
\end{aligned}
$$

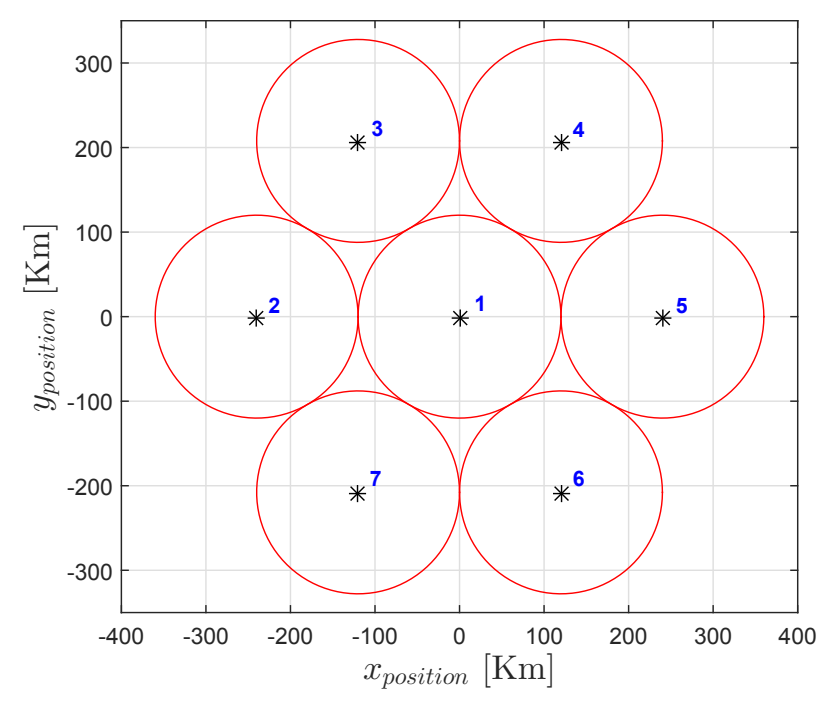

Figure 4: Beam pattern (circles) considered in the simulations, together with the position of the users (marked with the stars).

where $P_{\text {Tot }}$ represents the total available power at the transmitter.

The presented results are obtained assuming a 7-beam satellite channel based on the radiation pattern described by (3), with a maximum power gain $G_{\max }=10 \mathrm{~dB}$. The number of users $K$ is assumed to be equal to the number of transmitting antennas $N_{t}$, and the position of each user is fixed in the center of the respective beam. A picture of the considered beam pattern, together with the users position, is given in Fig. 4. The considered modulation is 8-PSK.

In Fig. 5 the attained minimum SINR (across the users) is shown as a function of the total available transmit power, for the proposed PAC approach and for the SPC approach. For fair comparison, the total available power is equally distributed amongst the transmit antennas when the PACs are considered, thus the value of $P_{\text {Th }}$ is set equal to $\frac{P_{\text {Tot }}}{N_{t}}$ (this assumption is kept for all the following comparisons). Moreover, the result is obtained by averaging over a large number of transmitted symbols. It is clearly visible how the attained SINR over a linear channel is lower with the proposed approach, showing a worse performance with respect to the SPC case. This could be expected considering that the use of PACs imply a more restrained exploitation of the available power, with a resulting lower SINR. In this regard, Fig. 6 the instantaneous power utilization for each transmit antenna is shown for the two approaches at hand, for a specific symbol slot. The total available power is fixed to 10 Watts. It is evident how the available power is not fully utilized with the proposed CIMMPAC approach, since the PACs are not attained for each transmit antenna.

Although this can be seen as a disadvantage of the proposed technique, it should be highlighted how the PACs are not avoidable in per-antenna power limited systems, where the 


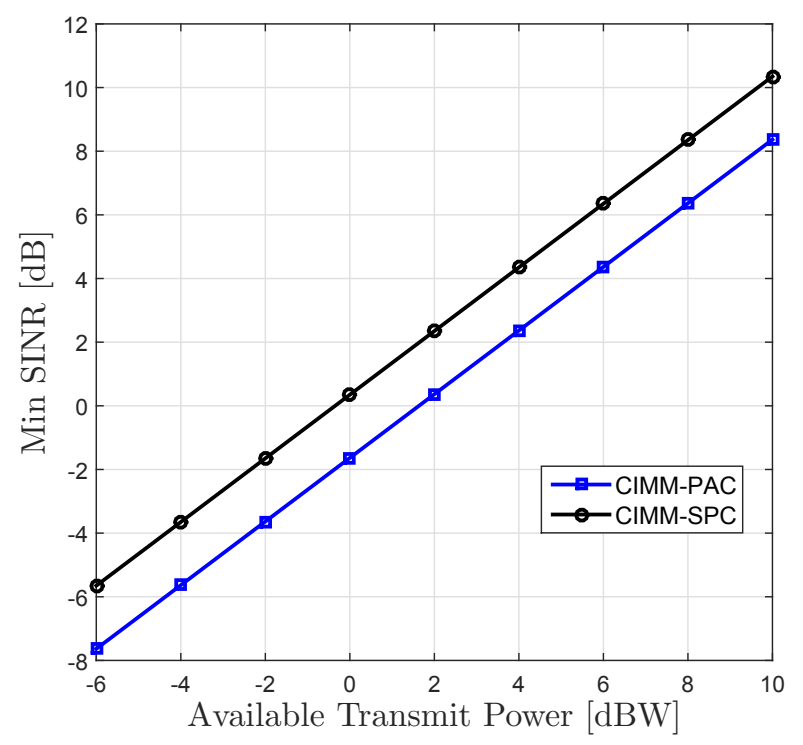

Figure 5: Minimum SINR amongst the users, in $\mathrm{dB}$, vs. available transmit power, in dBW.

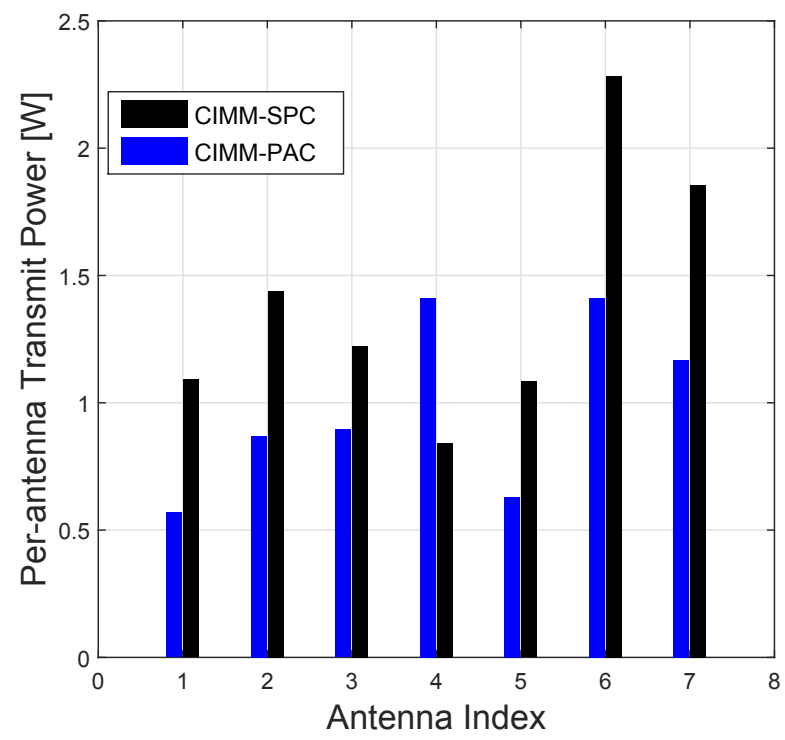

Figure 6: Per-antenna power utilization in Watts for a specific data information vector.

energy sharing between the different transmission chains is not possible. Furthermore, the advantages of the CIMM-PAC technique can be noticed focusing on non-linear satellite channels, accounting the non-linear characteristic of the TWTAs, as shown in Fig. 3. In fact, as already mentioned, in this case the existence of power peaks in the transmitted waveforms can determine a distortion effect with a considerable loss in performance. Focusing on this aspect, we show now how the proposed scheme affect the power distribution of the transmitted waveforms.

First of all, it is worth comparing the scatter plots of

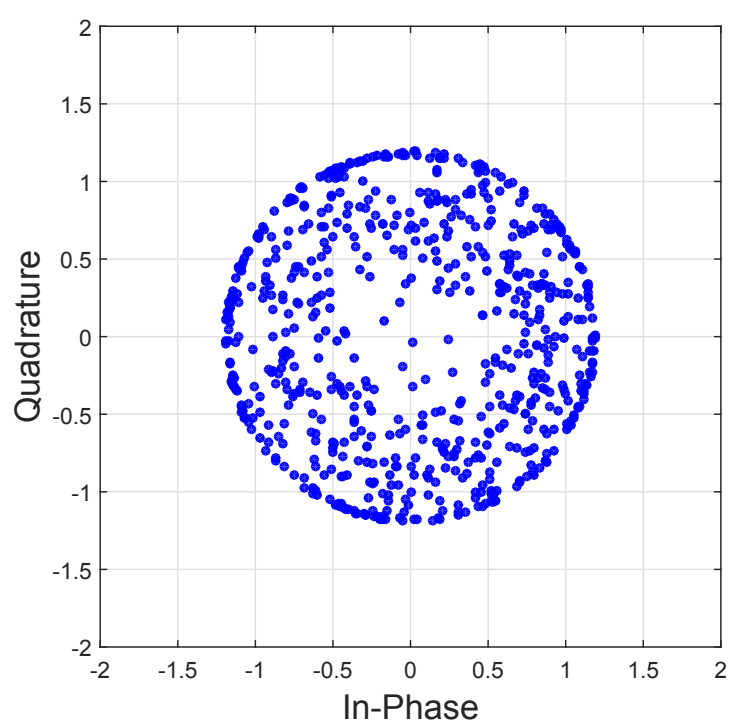

Figure 7: Scatter plot of the symbols associated to one antenna, after symbol-level precoding with PAC.

the symbols transmitted by one of the antennas, with the SPC approach, as shown in Fig. 2, and with the proposed PAC approach, as shown in Fig. 7. In both cases, a total available power of $10 \mathrm{dBW}$ is assumed. It is evident how the proposed CIMM-PAC scheme prevents the power peaks, which are observable in the CIMM-SPC case, where the perantenna power cannot be controlled. The proposed scheme, by imposing the PACs in the design of the transmitted symbols, results in a constellation lying inside a circle, and this clearly constitutes a relevant advantage with respect to the nonlinearities of the satellite channel.

In Fig. 8 the power transmitted by one of the antennas is considered (we take, without loss of generality, the antenna with indexed by 1 , whose transmit power is $P_{1}=\left|x_{1}\right|^{2}$ ), and the empirical evaluation of its complementary cumulative distribution function (CCDF) is drawn for the CIMM-PAC and the CIMM-SPC approaches, assuming a total available power of $10 \mathrm{dBW}$ in both cases. The CCDF of the power $P_{1}$ is a function of a variable $z$ defined as the probability of $P_{1}$ being larger than $z$, i.e., $\mathrm{CCDF}_{P_{1}}(z)=\operatorname{Pr}\left(P_{1}>z\right)$. With respect to the SPC case, where a long tail is visible in the curve, in the proposed approach the power results to be more bounded. The difference between the maximum value of $P_{1}$ in the two cases is over $4 \mathrm{dBW}$, which is considerable taking into account the saturation effects of non-linear TWTAs.

Another interesting figure of merit for evaluating the dynamic properties of the transmitted waveforms, with respect to the non-linear satellite channel, is the instantaneous-toaverage power ratio (IAPR). This quantity, referring again to the antenna indexed by 1 , is defined as:

$$
\operatorname{IAPR}_{1}=\frac{\left|x_{1}\right|^{2}}{\mathbb{E}_{n}\left|x_{1}\right|^{2}}
$$




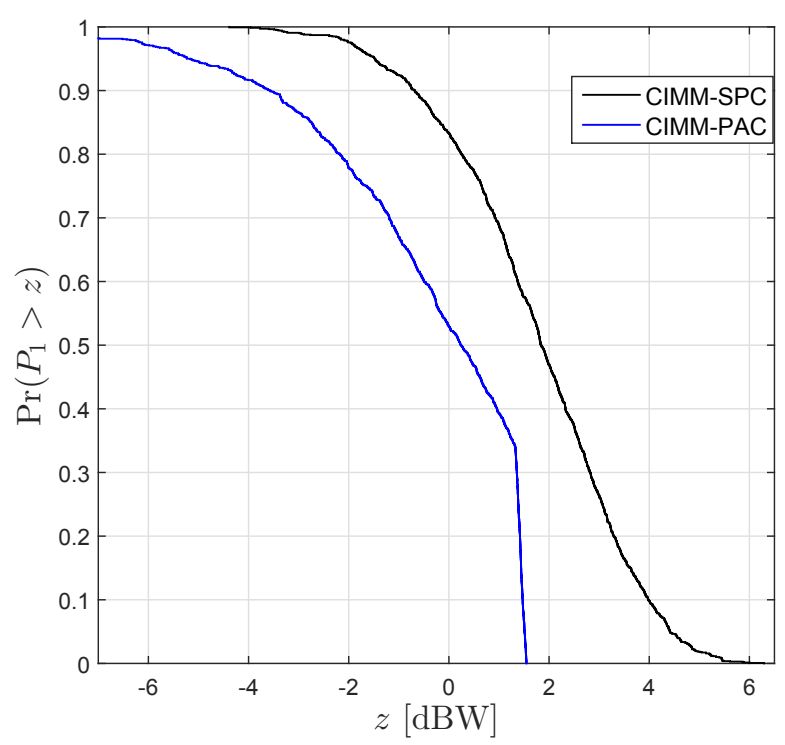

Figure 8: CCDF of the transmitted power by a single antenna.

and its maximum value over a certain time interval is the more common peak-to-average power ratio (PAPR). The IAPR distribution over time gives an information about the power variation around its average value, and thus about the power peaks. In Fig. 9, the empirical evaluation of the CCDF of the IAPR, for a single transmit antenna, is shown, considering the PAC and SPC cases. The total available power is again fixed to $10 \mathrm{dBW}$. The result shows how, with the proposed CIMM-PAC approach, also the IAPR is more bounded. This implies smaller variations of the transmitted power in time, and this property is very important for non-linear systems. A considerable difference of over $2 \mathrm{~dB}$ in the PAPR between the two techniques is observable.

\section{CONCLUSIONS}

In this work a novel technique for symbol-level precoding has been proposed, taking into account the per-antenna power limitations that arise typically in satellite systems. In particular, a max-min fair scheme, under per-antenna power constraints, is formalized and solved for M-PSK modulations. The solution has been carried out by relating the max-min fair problem to an equivalent per-antenna power minimization problem, and applying a bisection procedure. The performance of the technique has been assessed through simulation results, which show how the proposed scheme allows to reduce the power peaks of the transmitted waveforms. This feature makes the per-antenna constrained design suitable for systems corrupted by non-linear effects, such as satellite ones, where the power peaks reduction, and more in general the control on the transmitted power, implies relevant benefits.

\section{ACKNOWLEDGMENT}

This work was partially supported by the National Research Fund, Luxembourg, under the projects SATSENT, SeMiGod,

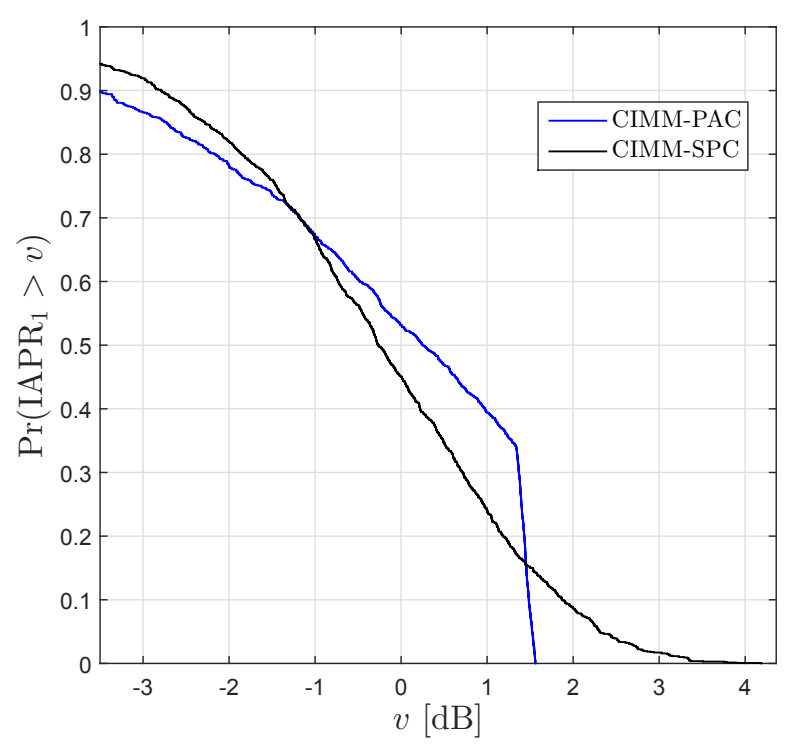

Figure 9: CCDF of the IAPR for a single transmit antenna.

and BroadSat (AFR project).

\section{REFERENCES}

[1] R. Roy and B. Ottersten, "Spatial division multiple access wireless communication systems," June 1997, US Patent 5,642,353. [Online]. Available: https://www.google.com/patents/US5642353

[2] J. D. Gayrard, "Terabit satellite: Myth or reality?" in Advances in Satellite and Space Communications, 2009. SPACOMM 2009. First International Conference on, July 2009, pp. 1-6.

[3] D. Mignolo, R. Emiliano, A. Ginesi, A. B. Alamanac, P. Angeletti, and M. Harverson, "Approaching terabit/s satellite capacity: A system analysis," in Proc. Ka Broadband Conf., Oct. 2011.

[4] B. Evans and P. Thompson, "Key issues and technologies for a terabit/s satellite," in 28th AIAA International Communications Satellite Systems Conference (ICSSC-2010), 2010.

[5] P. Thompson, B. Evans, L. Castenet, M. Bousquet, and T. Mathiopoulos, "Concepts and technologies for a terabit/s satellite," in SPACOMM 2011, The Third International Conference on Advances in Satellite and Space Communications, 2011, pp. 12-19.

[6] O. Vidal, G. Verelst, J. Lacan, E. Alberty, J. Radzik, and M. Bousquet, "Next generation high throughput satellite system," in Satellite Telecommunications (ESTEL), 2012 IEEE First AESS European Conference on, Oct 2012, pp. 1-7.

[7] Y.-F. Liu, Y.-H. Dai, and Z.-Q. Luo, "Coordinated beamforming for MISO interference channel: Complexity analysis and efficient algorithms," IEEE Transactions on Signal Processing, vol. 59, no. 3, pp. 1142-1157, 2011.

[8] E. Björnson, M. Bengtsson, and B. Ottersten, "Optimal multiuser transmit beamforming: A difficult problem with a simple solution structure [lecture notes]," IEEE Signal Processing Magazine, vol. 31, no. 4, pp. 142-148, July 2014.

[9] A. B. Gershman, N. D. Sidiropoulos, S. Shahbazpanahi, M. Bengtsson, and B. Ottersten, "Convex optimization-based beamforming," IEEE Signal Processing Magazine, vol. 27, no. 3, pp. 62-75, May 2010.

[10] M. Bengtsson and B. Ottersten, "Optimal and suboptimal transmit beamforming," in Handbook of Antennas in Wireless Communications. CRC Press, 2001, pp. 18-1-18-33, qC 20111107.

[11] M. Schubert and H. Boche, "Solution of the multiuser downlink beamforming problem with individual sinr constraints," IEEE Transactions on Vehicular Technology, vol. 53, no. 1, pp. 18-28, Jan 2004.

[12] M. Bengtsson and B. Ottersten, "Optimal downlink beamforming using semidefinite optimization," in Proc. of Annual Allert. Conf. on Commun. Control and Computing, vol. 37. Citeseer, 1999, pp. 987-996. 
[13] DVB Blue Book A83-2, "Second generation framing structure, channel coding and modulation systems for broadcasting, interactive services, news gathering and other broadband satellite applications; part II: S2extensions (S2X),

[14] W. Yu and T. Lan, "Transmitter optimization for the multi-antenna downlink with per-antenna power constraints," IEEE Transactions on Signal Processing, vol. 55, no. 6, pp. 2646-2660, June 2007

[15] G. Dartmann, X. Gong, W. Afzal, and G. Ascheid, "On the duality of the max min beamforming problem with per-antenna and per-antennaarray power constraints," IEEE Transactions on Vehicular Technology, vol. 62, no. 2, pp. 606-619, Feb 2013.

[16] G. Zheng, S. Chatzinotas, and B. Ottersten, "Generic optimization of linear precoding in multibeam satellite systems," IEEE Transactions on Wireless Communications, vol. 11, no. 6, pp. 2308-2320, June 2012.

[17] D. Christopoulos, S. Chatzinotas, and B. Ottersten, "Weighted fair multicast multigroup beamforming under per-antenna power constraints," IEEE Transactions on Signal Processing, vol. 62, no. 19, pp. 5132 5142 , Oct 2014.

[18] C. Masouros and E. Alsusa, "Dynamic linear precoding for the exploitation of known interference in mimo broadcast systems," IEEE Transactions on Wireless Communications, vol. 8, no. 3, pp. 1396-1404, March 2009.

[19] C. Masouros, "Correlation rotation linear precoding for mimo broadcast communications," IEEE Transactions on Signal Processing, vol. 59, no. 1, pp. $252-262$, Jan. 2011.

[20] C. Masouros and G. Zheng, "Exploiting known interference as green signal power for downlink beamforming optimization," IEEE Transactions on Signal Processing, vol. 63, no. 14, pp. 3628-3640, July 2015.

[21] M. Alodeh, S. Chatzinotas, and B. Ottersten, "Energy-efficient symbollevel precoding in multiuser miso based on relaxed detection region,' IEEE Transactions on Wireless Communications, vol. PP, no. 99, pp. $1-1,2016$.

[22] M. Alodeh, S. Chatzinotas, and B. Ottersten, "Constructive multiuse interference in symbol level precoding for the miso downlink channel,' IEEE Transactions on Signal Processing,, vol. 63, no. 9, pp. 2239-2252, May 2015.

[23] M. Alodeh, S. Chatzinotas, and B. Ottersten, "Constructive interference through symbol level precoding for multi-level modulation," in 2015 IEEE Global Communications Conference (GLOBECOM), Dec 2015, pp. 1-6.

[24] D. Christopoulos, P.-D. Arapoglou, S. Chatzinotas, and B. Ottersten, "Linear precoding in multibeam satcoms: Practical constraints," in 31st AIAA International Communications Satellite Systems Conference (ICSSC), Florence, IT, Oct. 2013.

[25] E. Casini, R. D. Gaudenzi, and A. Ginesi, "DVB-S2 modem algorithms design and performance over typical satellite channels," International Journal of Sat. Comm. and Netw., vol. 22, no. 3, pp. 281-318, 2004. [Online]. Available: http://dx.doi.org/10.1002/sat.791

[26] D. Spano, D. Christopoulos, S. Andrenacci, S. Chatzinotas, J. Krause, and B. Ottersten, "Total degradation analysis of precoded signals onto non-linear satellite channels," in 21st Ka and Broadband Communications Conference, Oct 2015.

[27] ETSI EN 302307 V1.1.2, "Digital video broadcasting (DVB); second generation framing structure, channel coding and modulation systems for broadcasting, interactive services, news gathering and other broad-band satellite applications (DVB-S2), european broadcasting union (EBU)."

[28] G. Karam and H. Sari, "A data predistortion technique with memory for qam radio systems," IEEE Transactions on Communications, vol. 39, no. 2, pp. 336-344, 1991.

[29] R. Piazza, M. R. B. Shankar, and B. Ottersten, "Data predistortion for multicarrier satellite channels based on direct learning," IEEE Transactions on Signal Processing, vol. 62, no. 22, pp. 5868-5880, Nov 2014.

[30] A. Mengali, B. Shankar, and B. Ottersten, "Joint predistortion and papr reduction in multibeam satellite systems," in IEEE International Conference on Communications (ICC 2016), Kuala Lumpur, Malaysia, May 2016, accepted.

[31] M. Diaz, N. Courville, C. Mosquera, G. Liva, and G. Corazza, "Nonlinear interference mitigation for broadband multimedia satellite systems," in Proc. Int. Work. Sat. Space Commun. (IWSSC), Sept. 2007, pp. 61-65.

[32] D. Spano, M. Alodeh, S. Chatzinotas, and B. Ottersten, "Per-antenna power minimization in symbol level precoding," in 2016 IEEE Global
Communications Conference (GLOBECOM), Washington DC, USA, Dec 2016, submitted.

[33] A. Kalantari, M. Soltanalian, S. Maleki, S. Chatzinotas, and B. Ottersten, "Secure m-psk communication via directional modulation," in Acoustics, Speech, and Signal Processing, 2016. Proceedings. (ICASSP '16). IEEE International Conference on, March 2016.

[34] S. Boyd and L. Vandenberghe, Convex optimization. Cambridge Univ. Press, 2004 